\title{
Location of an autosomal factor causing sterility in Drosophila mojavensis males carrying the Drosophila arizonensis $\mathrm{Y}$ chromosome
}

\author{
A. C. Pantazidis* and \\ E. Zouros*†
}

\author{
* Department of Biology, University of Crete, and \\ Institute of Molecular Biology and Biotechnology, \\ Research Center of Crete, Iraklion, Crete, Greece. \\ $\dagger$ Department of Biology, Dalhousie University, \\ Halifax, N.S., Canada B3H 4J1.
}

\begin{abstract}
Drosophila mojavensis males whose $Y$ chromosome is replaced by the $Y$ of $D$. arizonensis, have immotile sperm. Sperm motility is restored if one member of the fourth autosome pair is also replaced by an arizonensis homologue. We present evidence that the effect of the fourth chromosome is due to a single Mendelian factor and map this factor relative to the two markers available for this chromosome. This is an essential first step towards understanding the nature of the incompatibility between the arizonensis $Y$ chromosome and the mojavensis fourth chromosome responsible for this type of post-zygotic isolation between these closely related species.
\end{abstract}

\section{INTRODUCTION}

Hybrid sterility or inviability has been recognised as one of the first reproductive isolating mechanisms appearing between two diverging populations, and has, for this reason, received special attention from the students of speciation. The genetic basis of post-zygotic mechanisms of reproductive isolation has been studied in particular detail in Drosophila, where the accumulated evidence suggests the existence of incompatibilities between heterospecific sex chromosomes or sex chromosomes and autosomes, and the involvement of several genetic factors (Dobzhansky, 1936; Sturtevant and Novitski, 1941; Mainland, 1941, Ehrman, 1963; Prakash, 1972; Dobzhansky, 1974; Schafer, 1978; Coyne, 1984; Coyne, 1985; Naveira and Fontdevila, 1986; Vigneault and Zouros, 1986). Further understanding of the mechanisms leading to reproductive isolation between different yet closely related species requires a deeper knowledge of the function of the particular genes involved. The first step in this direction is the identification and the mapping of these genes. There already exist three examples of this type of studies. Watanabe (1979) has described a mutant

Address for Correspondence: Dr. E. Zouros, Department of Biology, Dalhousie University, Halifax, N.S., Canada, B3H 4J1. in the chromosomal arm 2R of Drosophila simulans that when crossed to $D$. melanogaster allows the survival to adulthood of progeny of both sexes. Coyne and Charlesworth (1986) have mapped an $\mathrm{X}$-linked factor in $D$. simulans that causes sterility in male hybrids with $D$. mauritiana. Most recently, Hutter and Ashburner (1987) reported an X-linked mutation in $D$. melanogaster that rescues the normally lethal hybrid males from crosses of this species with its close relatives $D$. simulans, $D$. mauritiana and $D$. sechellia.

Zouros and his associates have been studying the genetic basis of male hybrid sterility in the sibling species-pair $D$. mojavensis and $D$. arizonensis (Zouros, 1981; Vigneault and Zouros, 1986; Zouros et al., in press). In this species-pair hybrids are viable and fertile except males from the cross $D$. mojavensis male $\times D$. arizonensis female which are sterile. Several incompatibilities have been identified between sex chromosomes and autosomes that may cause sperm immotility in males from different backcrosses. Vigneault and Zouros (1986) have shown that males whose Y chromosome is of arizonensis origin but are mojavensis in any other respect (egg cytoplasm, X chromosome and all autosomes) are sterile, and that fertility can be restored if one member of the fourth autosome pair is replaced with an arizonensis homologue. This provides one of the 
simplest possible types of chromosomal interaction restoring fertility in hybrids. As a first step to the study of this interaction we have obtained data suggesting that the effect of the fourth chromosome is attributable to a single Mendelian factor (we refer to this and any other factor that may affect sperm motility as "sperm motility factor", SMF) and have mapped it in relation to two markers available for this chromosome.

\section{MATERIALS AND METHODS}

We have used a mojavensis stock, donated by Dr T. Starmer, fixed for a recessive autosomal mutant that causes brown-coloured eyes instead of the wild-type red. We refer to this stock as $b r$ and the wild-type as $b r^{+}$. The arizonensis stock used was A875, a gift from Dr W. Heed. The two stocks are fixed for different electromorphs of enzyme loci marking the $\mathrm{X}$ chromosome and each autosome except the small dot-like sixth chromosome. These markers, the electrophoretic assays for their scoring and the assay for scoring sperm motility are described in Vigneault and Zouros (1986). We use the following symbolism: X, Y, II to VI to refer to $\mathrm{X}, \mathrm{Y}$ and second to sixth chromosomes; $A$ to refer to a complete haploid complement of autosomes; $a, m$ and $r$ to refer to the specific origin of a chromosome (arizonensis, mojavensis or recombinant, respectively).

\section{RESULTS}

\section{Location of the br mutation on the fourth chromosome}

To locate the $b r$ mutation, females from the $b r$ strain were crossed to arizonensis A875. Males from this cross were crossed to $b r$ females. Males from this first backcross (named BC-1) of both the $b r$ and $b r^{+}$phenotype were individually crossed to $b r$. Only one cross out of four produced progeny. After electrophoresis, the male parent of this fertile cross was found to be homozygous for the mojavensis marker of chromosomes III (malic dehydrogenase, MDH) and V (peptidase-2, PEP2 ), and heterozygous for the markers of chromosomes II (octanol dehydrogenase, ODH) and IV (phosphoglucose mutase, PGM); it was also $\mathrm{br}^{+}$. Half of this progeny (BC-2) were $b r$ and half were of $\mathrm{br}^{+}$. Twelve males from each phenotype were individually crossed to $b r . b r$ fathers produced no offspring and upon examination they were all found to carry immotile sperm; they also were homozygous for the mojavensis PGM allozyme. All crosses with $b r^{+}$males produced offspring and after electrophoresis they were found to be heterozygous for PGM. Both $b r^{+}$and $b r$ male classes contain mojavensis homozygotes and heterozygotes for $\mathrm{ODH}$. These results place the $b r$ locus on the fourth chromosome. $b r^{+}$males emerging from cultures whose the male parents were homozygous for $\mathrm{ODH}$ were pooled together and mass-crossed to $b r$. These $\mathrm{BC}-3$ males were homozygous for the mojavensis homologue of chromosomes II, III and V and heterozygous for $\mathrm{IV}$; they were also $\mathrm{Xm} \mathrm{Ya}$ regarding the sex chromosomes and of unknown condition ( $\mathrm{mm}$ or $m a)$ for the unmarked sixth chromosome. This type of male, to which we refer as Xm YaIVam, is being maintained by continued backcrossing to $b r$. Experiments reported here were performed in about the twentieth generation of backcrossing.

\section{Chromosomal control of sperm motility in XmYalVam}

The first report (Zouros, 1981) of sperm immotility in males resulting from the backcross of $\mathrm{X} m \mathrm{YaAam}$ to mojavensis female implicated only the $Y$ and the fourth chromosome. In a larger study of the same phenomenon Vigneault and Zouros (1986) confirmed the involvement of these two chromosomes, but a complication arose concerning the third chromosome: whereas males of the type X $m$ YaIII am IVam had always motile sperm, males of the type XmYaIIImm IVam sometimes had motile and sometimes immotile sperm. One hypothesis entertained by Vigneault and Zouros (1986) was the involvement of the sixth chromosome. The hypothesis suggests that chromosomes III and VI carry duplicate copies of the same SMF (the two genes need only be "functional" duplicates) and that sperm motility in Xm YaIVam males requires heterozygosity for either the third or the sixth chromosome. Upon repeated backcrossing to mojavensis females there is a 50 per cent chance that the line will be fixed for the mojavensis homologue of the sixth chromosome and 50 per cent of the third. In the former case all males of the type XmYaIIImm IVam will have immotile sperm (they will lack both copies of $\mathrm{SMF}$ ); in the latter case half of these males will have immotile sperm (these will be VI $\mathrm{mm}$ ) and half will have motile sperm (these will be VI $a m$ ).

There is a simple way to rule out the involvement of the sixth chromosome in our backcrosses. Because the single $\mathrm{BC}-1$ male from which we 
started the line was fixed for the third mojavensis chromosome, the sixth chromosome hypothesis necessitates that fertile males are heterozygous for the sixth chromosome and that in each generation half of the heterozygotes for the fourth chromosome (which phenotypically are $b^{+}$) will have immotile sperm (these will be IVamVI $m m$ ). We have repeatedly checked this prediction and found it wrong: all $b r^{+}$males regardless of generation had motile sperm and all $b r$ had immotile sperm.

\section{Mapping of the fourth chromosome SMF relative to $\mathrm{br}$ and $P G M$}

The exclusion of the sixth chromosome hypothesis means that the fertile males from our repeated backcrosses are mojavensis in all respects except the $\mathrm{Y}$ chromosome and one fourth chromosome which are of arizonensis origin (the chance that the sixth arizonensis chromosome is present in the twentieth backcross is $0 \cdot 5^{20} \simeq 0$ ). Our next step was to map the SMF of the fourth chromosome relative to the two markers available for this chromosome, $b r$ and PMG. We note that the fourth chromosome of mojavensis is analogous to $3 \mathrm{~L}$ of $D$. melanogaster and XR of D. pseudoobscura (Vigneault and Zouros, 1986) and that mojavensis and arizonensis are homosequential for this chromosome (Wasserman, 1962). We, therefore, expect free recombination in females heterospecific for this chromosome. The only cross that could provide this mapping information is between $b r^{+}$males and females from our backcrosses. This cross is of the type: $\mathrm{X} m \mathrm{X} m \mathrm{IV} a m \times \mathrm{X} m \mathrm{Y} a \mathrm{IV} a m$, with all chromosomes not shown of mojavensis origin. Heterospecificity for the fourth chromosome is needed in the female to provide the recombinant genotypes, and in the male to provide fertility. The male should also carry the $\mathrm{Y} a$ so that the effect of the fourth chromosome SMF (which is expressed as an interaction with $\mathrm{Ya}$ ) can be detected among the offspring.

There are 16 genotypic classes among male progeny from this cross (table 1). Eight of these (part B of table 1) correspond to products of a test-cross (their paternal gamete carries the recessive alleles at all loci to be mapped), but one parental class (class B1) cannot be phenotypically separated from other genotypes resulting from classes in part A. Among females (table 2) there are eight genotypic classes of which four correspond to a back-cross (part B of table 2), and again one parental class (B1) cannot be separated from other classes. To obtain a relatively large sample of progeny and also to check the repeatability of the results we performed two separate crosses. The raw data are given in tables 1 and 2 . The data sets from the two experiments are highly homogeneous (for males, the chi-square is 1.61 on 8 degrees of freedom, $0.990<P<0.995$; for females 1.17 on 4 degrees of freedom, $0.75<P<0.90$ ), so further analysis is performed on the pooled data (third number in tables 1 and 2).

A careful inspection of tables 1 and 2 reveals large deviations of single-locus genotypes from Mendelian expectations. These deviations do not,

Table 1 Male progeny from two mapping crosses of the type X $m$ X $m$ IV am $\times$ X $m$ YaIVam. All other chromosomes are of mojavensis origin. The first number in each class is the result from the first cross, the second from the second cross. Phenotype I is $\mathrm{br}^{+}$, motile sperm and PGMaa; phenotype II is $\mathrm{br}^{+}$, motile sperm and PGMam; classes B2 to B8 have their own phenotypes

\begin{tabular}{|c|c|c|}
\hline \multirow[b]{2}{*}{ Female gametes } & \multicolumn{2}{|l|}{ Male gametes } \\
\hline & A. $b r^{+} S M F a P G M a$ & B. br SMFm PGMm \\
\hline 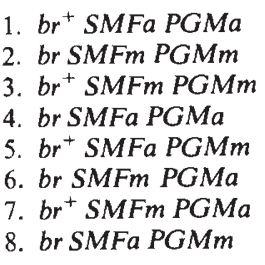 & $\begin{array}{l}\text { Phen. I } \\
\text { Phen. II } \\
\text { Phen. II } \\
\text { Phen. I } \\
\text { Phen. II } \\
\text { Phen. I } \\
\text { Phen. I } \\
\text { Phen. II }\end{array}$ & $\begin{array}{l}\text { Phen. II } \\
59+40=99 \\
33+27=60 \\
27+23=50 \\
34+20=54 \\
29+23=52 \\
16+12=28 \\
11+9=20\end{array}$ \\
\hline $\begin{array}{l}\text { Phenotype I } \\
\text { Phenotype II } \\
\text { Other phenotypes }\end{array}$ & $\begin{array}{r}54+36=90 \\
123+93=216 \\
363\end{array}$ & \\
\hline Total progeny & 669 & \\
\hline
\end{tabular}


Table 2 Female progeny from the two mapping crosses of table 1. Notation as in table 1 .

\begin{tabular}{lll}
\hline & \multicolumn{2}{c}{ Male gametes } \\
\cline { 2 - 3 } Female gametes & A. $b r^{+} P G M a$ & B. $b r$ PGMm \\
\hline 1. $b r^{+} P G M a$ & Phen. I & Phen. II \\
2. $b r P G M m$ & Phen. II & $66+62=128$ \\
3. $b r^{+} P G M m$ & Phen. II & $64+64=128$ \\
4. $b r P G M a$ & Phen. I & \\
Phenotype I & $52+42=94$ & \\
Phenotype II & $135+110=245$ & \\
Other phenotypes & & 370 \\
Total progeny & 709 & \\
\hline
\end{tabular}

however, exist in the subset of data resulting from the classes in the second column of tables 1 and 2 . To illustrate these points we give in table $3 \mathrm{~A}$ the observed numbers of single-locus phenotypes for the complete data set, and in table $3 \mathrm{~B}$ the numbers from classes B3 to B8 of table 1, and B3 plus B4 of table 2. Classes B2 had to be ignored because their symmetrical classes (B1) cannot be recovered. Focusing first on Table $3 \mathrm{~A}$ we see that the $b r^{+}$to $b r$ ratio deviates from the expected $3: 1$ ratio in favor of $b r$, but the ratio is the same in the two sexes (chi-square 1.437 on one degree of freedom, $0 \cdot 10<P<0 \cdot 25)$, thus the viability depression of $b r^{+}$is independent from sex. These calculations can be repeated for the other two loci. Given that sperm immotility occurs only if the male is homozygous for the mojavensis fourth chromosome, the expected ratio of motile to immotile is again $3: 1$, but the observed numbers deviate in favour of males with immotile sperm, i.e., again in the direction of the mojavensis homozygote. For the PGM locus the expected ratio is $1: 2: 1$ and the observed numbers again deviate in favor of the mojavensis homozygote, but the ratio is the same in the two sexes (chi-square $0 \cdot 11$ on two degrees of freedom, $0.90<P<0.95$ ), so again viability differences among genotypes are independent from sex. Turning now to table $3 \mathrm{~B}$ we see that the observed ratios closely conform to the expected $1: 1$ ratio for all three loci in both sexes.

The observation that there are no viability differences among the genotype classes in the second parts of tables 1 and 2 means that the information in these classes can be used for mapping purposes. It raises, however, the question of what causes the depression of viability in the first parts of the tables. We suggest that loss of viability is not the result of the mere presence of some arizonensis genetic material in an otherwise mojavensis background. Rather, the loss results

Table 3 One-locus genotypes from the data in Tables 1 and 2. MS: motile sperm, IS: immotile sperm. In part A tests for $b r^{+} / b r$ and $M S / I S$ are for departure from the $3: 1$ ratio and for $P G M$ from the $1: 2: 1$ ratio; all tests are significant at the 0.001 level. In part $B$ all tests are for departure from the $1: 1$ ratio; none of these tests is significant

\begin{tabular}{|c|c|c|c|c|c|c|}
\hline \multirow[b]{2}{*}{ Genotype } & \multicolumn{3}{|c|}{ A. All data } & \multicolumn{3}{|c|}{$\begin{array}{l}\text { B. Classes B3 to B8 (table 1) } \\
\text { and B3+B1 (table 2) }\end{array}$} \\
\hline & Males & Females & Total & Males & Females & Total \\
\hline$b r^{+}$ & 448 & 453 & 901 & 142 & 114 & 256 \\
\hline$b r_{2}$ & 221 & 256 & 477 & 122 & 128 & 250 \\
\hline$x^{2}$ & $23 \cdot 03$ & $46 \cdot 65$ & $67 \cdot 95$ & $1 \cdot 52$ & 0.81 & 0.07 \\
\hline PGMaa & 90 & 94 & 184 & - & - & - \\
\hline PGMam & 346 & 373 & 719 & 130 & 128 & 258 \\
\hline$P G M m m$ & 233 & 242 & 475 & 134 & 114 & 248 \\
\hline$x^{2}$ & 61.91 & $63 \cdot 72$ & $125 \cdot 46$ & 0.06 & 0.81 & $0 \cdot 20$ \\
\hline$M S$ & 430 & - & - & 124 & - & - \\
\hline IS & 239 & - & - & 140 & - & - \\
\hline$x^{2}$ & $41 \cdot 04$ & & & 0.97 & & \\
\hline
\end{tabular}


from homozygosity for arizonensis material for some parts of the genome. Alternatively, we can think of the viability depression as the result of segmental nullosomy for mojavensis material in a mojavensis genome. We note that all classes of the second part of tables 1 and 2 have inherited a complete (non-recombinant) mojavensis chromosome from their father and therefore cannot be nullosomic for any part of their genome. To obtain further evidence for this hypothesis we counted the offspring from one large backcross of the type routinely used for the maintenance of X $m$ YaIVam males. The results are shown in table 4 . We see that the number of $\mathrm{br}^{+}$males or females (which are also heterozygous for PGM and, for males, with motile sperm) is not statistically different from the number of $b r$ (which are homozygous for PGM and, for males, with immotile sperm). These individuals carry no recombinant chromosomes and cannot be nullosomic for mojavensis material in any part of their genome.

We are now in a position to use the data of table 1 for mapping. One way to do this is to obtain first an estimate for the missing class B1. The data of table 4 indicate that this class must be equal to class B2; thus, the best estimate for it is 99 . We now have all eight classes of the test-cross which places the SMF almost exactly in the middle between the two markers, with the distance from $b r$ at $34 \cdot 2+2 \cdot 5$ (one standard error) centimorgans, and from PGM at $33 \cdot 3+2 \cdot 5$. A more involved estimation of recombination distances makes use of all data of table 1 . To do this we need to take into account the reduced viabilities of the classes in the first half of the table. Because we have shown that viability depression is independent from sex (table 3 ), we can obtain viability estimates from the females (table 2) and used them as independent estimates to derive maximum likelihood estimates of recombination distances from the males (table 1). The expected number of class B1 of table 2 is 128 (the same as B2). If there was no viability depression, the total number in part $A$ of table 2 ought to be the same as in part B, i.e., 498. Also,

Table 4 Progeny from the cross X $m X m I V m m \times X m$ Y $a \mathrm{IV} a m$. All other chromosomes are of mojavensis origin. The tests are for departure from the $1: 1$ ratio; none of them is significant

\begin{tabular}{lccc}
\hline Genotype & Males & Females & Total \\
\hline$b r^{+}$ & 180 & 189 & 369 \\
$b r$ & 190 & 174 & 364 \\
$\chi^{2}$ & 0.27 & 0.62 & 0.03 \\
\hline
\end{tabular}

the sum of classes A1 and A4 ( $b r^{+}$and homozygous for arizonensis PGM) should equal the sum of B1 and B4, i.e., 256. The observed number is 94 which gives a viability estimate for this phenotype of $94 / 256=0 \cdot 367$. For the same reason the combined number of classes A2 and A3 $\left(b r r^{+}\right.$and heterozygous for PGM) should equal the sum of classes $\mathrm{B} 2$ and B3, i.e., 242, but the observed number must be 117 (total observed 245 minus 128 that must belong to class $\mathrm{B} 1$ ). This gives a viability estimate for this phenotype of $117 / 242=0 \cdot 483$. We can now write the likelihood function for the data of table 1. If $r_{1}$ is the recombination frequency between $b r$ and SMF, and $r_{2}$ between SMF and PGM, the zygotic frequencies of each of the 16 classes can be written in terms of $r_{1}$ and $r_{2}$. These must be multiplied by their viabilities, which are one for the eight classes of part B, 0.367 for classes A1, A4, A6 and A7, and 0.483 for the classes A2, A3, $\mathrm{A} 5$ and A8. The function to be maximised is:

$$
\begin{aligned}
L= & (1 / 4)^{669}(0 \cdot 367)^{90}\left[0 \cdot 483+\left(1-r_{1}\right)\left(1-r_{2}\right)\right]^{216} \\
& \cdot\left(1-r_{1}\right)^{205}\left(1-r_{2}\right)^{209} r_{1}^{158} r_{2}^{154} .
\end{aligned}
$$

The first partial derivatives of $\log L$ were set to zero and the resulting system in two unknowns was solved by numerical reiteration using Newton's method of steepest ascent. The resulting estimates are 33.99 for $r_{1}$ and 33.03 for $r_{2}$, which are very close to those obtained by the simpler method described above.

\section{The fourth chromosome SMF behaves as a single Mendelian factor}

We have so far assumed that sperm motility in $\mathrm{Xm} \mathrm{YaIVam}$ is caused by a single locus in the fourth chromosome. Because in our backcrosses the fourth chromosome is transmitted through the male as a block, the 1:1 segregation of the two types (table 4) does not distinguish between the one-locus hypothesis and the alternative hypothesis of several loci dispersed in that chromosome. To obtain evidence for one or the other hypothesis, the second hypothesis must be made more explicit. More specifically, there can be two basic alternatives about the mode of action of multiple factors: only one of these is sufficient to establish synergism with $\mathrm{Ya}$ and produce motile sperm or the whole set of them is needed. When recombinants of the fourth chromosome are recovered among male progeny, the first mode predicts that more than half of the males will have motile sperm; the second mode predicts less than half; an 1:1 ratio is consistent with the single-locus 
hypothesis. When this test was applied to classes B3 to B8 of table 1 produced no significant deviations from the $1: 1$ ratio (table $3 \mathrm{~B}$ ). Additional evidence that the SMF behaves as a single Mendelian factor can be gained from considering all males in table 1 . When we compare the observed motile/immotile ratio to $\mathrm{br}^{+} / \mathrm{br}$ or to (PGMaa+ PGMam)/PGMmm (these ratios are $430 / 239$, $448 / 221,436 / 233$, respectively; table 3 a) we find that they are not statistically different (chi-square 1.27 on two degrees of freedom, $0.50<P<0.75$ ). This suggests that the SMF behaves as a single locus, like $b r$ or $P G M$, and the deviation of motile/immotile from the expected $3: 1$ ratio can be attributed to viability depression.

\section{DISCUSSION}

We have presented evidence that sperm motility in $\mathrm{Xm}$ YaIVam males maintained through repeated backcrossing to br mojavensis is the result of a synergism between the arizonensis $\mathrm{Y}$ chromosome and a single factor on the fourth arizonensis chromosome. No other factors are involved. This does not mean that no other SMF exist in this pair of species, but simply that these other factors are compatible with normal spermatogenesis in an $\mathrm{X} m \mathrm{YaA} m m$ background. We cannot, of course, exclude the possibility of more than one closely linked factor on the fourth chromosome. The mapping data suggest that this factor (or cluster of factors) is located between $b r$ and PGM, about 33 centimorgans away from each. More detailed mapping is not possible at present because of paucity of markers. We can say very little about the nature of the interaction between the $\mathrm{Y}$ chromosome and the fourth chnromosome factor under normal (homospecific) or heterospecific conditions, except that is mostly likely trans-acting (one arizonensis chromosome suffices for sperm motility). Also, whatever are the $\mathrm{Y}$ chromosome genes involved in this interaction there should be no copies of them in the $\mathrm{X}$ chromosome, if the two sex chromosomes of mojavensis are found to contain homologies. This follows from the fact that $\mathrm{X} m \mathrm{YaIVmm}$ has immotile sperm. Goldstein et al (1982) have shown that $D$. melanogaster males deficient for different regions of their Y chromosome were missing several testis proteins of high molecular weight that are apparently structural components of the sperm axoneme. Our next step is to combine electron microscopy and protein studies of testis from $\mathrm{XmYa}$ males with and without the arizonensis SMF in the hope to learn more about the nature of this interspecific incompatibility.

Acknowledgments We thank Dr K. Lofdahl for the initial backcrosses that help us locate the $b r$ gene, DrV. Galanopoulos for discussions of the paper, and Dr P. Laloussis for the numerical solution of the likelihood function. A.C.P. is supported with a graduate scholarship from the Research Center of Crete, Greece. This is part of a research program supported by an operating grant from Natural Sciences and Engineering Council of Canada to E.Z.

\section{REFERENCES}

COYNE, J. A. 1984. Genetic basis of male sterility in hybrids between two closely related species of Drosophila. Proc. Nat. Acad. Sci. USA, 84, 4444-4447.

COYNE, J. A. 1985. The genetic basis of Haldane's rule. Nature, $314,736-738$.

COYNE, J. A. AND CHARLESWORTH, B. 1986. Location of an $\mathrm{X}$-linked factor causing sterility in male hybrids of Drosophila simulans and Drosophila mauritiana. Heredity, 57, 243-246.

DOBZHANSKY, TH. 1936. Studies of hybrid sterility. II. Localization of sterility factors in Drosophila pseudoobscura hybrids. Genetics, 21, 113-135.

DOBZHANSKY, TH. 1974. Genetic analysis of hybrid sterility within the species Drosophila pseudoobscura. Hereditas, 77 , $81-88$.

EHRMAN, L. 1963. Apparent cytoplasmic sterility in Drosophila paulistorum. Proc. Nat. Acad. Sci. USA, 49, 155-158.

GOLDSTEIN, L. S. B., HARDY, R. W. AND LINDSLEY, D. L. 1982. Structural genes of the $\mathrm{Y}$ chromosome of Drosophila melanogaster. Proc. Nat. Acad. Sci. USA, 79, 7405-7409.

HUTTER, P. AND ASHBURNER, M. 1987. Genetic rescue of inviable hybrids between Drosophila melanogaster and its sibling species. Nature, 327, 331-333.

MAINLAND, G. B. 1941. Studies in speciation. III. The Drosophila macrospina group. Genetics, 26, s160.

NAVEIRA, H. AND FONTDEVILA, A. 1986. The evolutionary history of Drosophila buzzatii. XII. The genetic basis of sterility in hybrids between $D$. buzzatii and its sibling $D$. serido from Argentina. Genetics, 114, 841-857.

PRAKASH, S. 1972. Origin of reproductive isolation in the absence of apparent genic differentiation in a geographic isolate of Drosophila pseudoobscura. Genetics, 72, 143-155.

SCHAFER, U. 1978. Sterility in Drosophila hydel $\times$ Drosophila neohydel hybrids. Genetica, 49, 205-214.

STURTEVANT, A. H. AND NOVITSKI, E. 1941. Sterility in crosses of geographical races of Drosophila micromelanica. Proc. Nat. Acad. Sci. USA, 27, 392-394.

VIGNEAULT, G. AND ZOUROS, E. 1986. The genetics of asymmetrical male sterility in Drosophila mojavensis and Drosophila arizonensis hybrids: interactions between the $\mathrm{Y}$ chromosome and autosomes, Evolution, 40, 1160-1170.

WATANABE, T. K. 1979. A gene that rescues the lethal hybrids between Drosophila melanogaster and Drosophila simulans. Jap. J. Genetics, 54, 325-331.

ZOUROS, E. 1981. An autosome-Y chromosome combination that causes sterility in Drosophila mojavensis $\times$ Drosophila arizonensis hybrids. Dros. Infor. Serv., 56, 167-168.

ZOUROS, E., LOFDAHL, K. AND MARTIN, P. A. Male hybrid sterility in Drosophila: interactions between autosomes and sex chromosomes in crosses of $D$. mojavensis and $D$. arizonensis. Evolution (in press). 